type, indicating that such adaptation is unique to each strain of $P$. aeruginosa and does not result from a single mechanism shared by the whole species.

FROM: Loughlin MF, Jones MV, Lambert PA. Pseudomonas aeruginosa cells adapted to benzalkonium chloride show resistance to other membrane-active agents but not to clinically relevant antibiotics. I Antimicrob Chemother 2002;49:631-639.

\section{Biofilms: Survival Mechanisms of Clinically Relevant Microorganisms}

Donlan and Costerton point out that although biofilms were first described by Antonie van Leeuwenhoek, the theory describing the biofilm process was not developed until 1978. It is now understood that biofilms are universal, occurring in aquatic and industrial water systems and in a large number of environments and medical devices relevant for public health. Using tools such as the scanning electron microscope and, more recently, the confocal laser scanning microscope, biofilm researchers now understand that biofilms are not unstructured, homogeneous deposits of cells and accumulated slime, but rather complex communities of surface-associated cells enclosed in a polymer matrix containing open water channels.

Further studies have shown that the biofilm phenotype can be described in terms of the genes expressed by biofilm-associated cells. Microorganisms growing in a biofilm are highly resistant to antimicrobial agents by one or more mechanisms. Biofilm-associated microorganisms have beerl shown to be associated with several diseases in humans, such as native valve endocarditis and cystic fibrosis, and to colonize a wide variety of medical devices. Although epidemiologic evidence points to biofilms as a source of several infectious diseases, the exact mechanisms by which biofilm-associated microorganisms elicit disease are poorly understood. Detachment of cells or cell aggregates, production of endotoxin, increased resistance to the host immune system, and provision of a niche for the generation of resistant organisms are all biofilm processes that could initiate the disease process. Effective strategies to prevent or control biofilms on medical devices must take into consideration the unique and tenacious nature of biofilms. Current intervention strategies are designed to prevent initial device colonization, minimize microbial cell attachment to the device, penetrate the biofilm matrix and kill the associated cells, or remove the device from the patient. In the future, treatments may be based on the inhibition of genes involved in cell attachment and biofilm formation.

FROM: Donlan RM, Costerton JW. Biofilms: survival mechanisms of clinically relevant microorganisms. Clin Microbiol Rev 2002;15:167-193.

\section{The Success of Routine, Voluntary Inpatient HIV Testing}

Despite current recommendations for human immunodeficiency virus (HIV) counseling and testing among patients admitted to hospitals with at least a $1 \%$ prevalence of HIV infection, an estimated 300,000 individuals in the United States remain unaware of their HIV infection. Walensky and colleagues implemented the Think HIV program, which offered voluntary HIV counseling and testing to patients admitted to the medical service of a Boston, Massachusetts, teaching hospital. The results of this effort were compared with testing results from a 15-month historical control period.

Patients admitted during the program period were 3.4 times more likely to undergo testing for HIV than were those admitted during the control period ( $95 \%$ confidence interval $\left[\mathrm{CI}_{95}\right], 2.8$ to 4.1 ). The testing program detected approximately two new diagnoses of HIV infection per month, compared with one per month during the control period. Patients who underwent testing during the program, and who likely would not have done so without this initiative, had an estimated prevalence of HIV infection of $3.8 \%\left(\mathrm{Cl}_{95}, 1.8 \%\right.$ to $\left.5.8 \%\right)$.

The authors concluded that testing efforts for HIV targeted at only symptomatic patients are inadequate to identify the one-third of HIV-seropositive individuals in the United States who are unaware of their infection. They point out that their results show that in a single urban hospital, voluntary, routine inpatient HIV counseling and testing was successful as a screening program by identifying a substantial number of patients with undiagnosed HIV. These patients then can be informed, counseled, and linked to care and treatment. Seventy-two hospitals nationwide have demographics similar to those of the study hospital, suggesting that these results are generalizable to many urban hospitals.

FROM: Walensky RP, Losina E, Steger-Craven KA, Freedberg KA. Identifying undiagnosed human immunodeficiency virus: the yield of routine, voluntary inpatient testing. Arch Intern Med 2002;162:887-892. 